\title{
Special Case Algorithm for N-jobs M-machines Flow Shop Scheduling Problems
}

\section{Geleta Tadele Mohammed}

Department of Mathematics, College of Natural Sciences, Arba Minch University, Arba Minch, Ethiopia

Email address:

geletatadele@gmail.com,geleta.tadele@amu.edu.et

\section{To cite this article:}

Geleta Tadele Mohammed. Special Case Algorithm for N-jobs M-machines Flow Shop Scheduling Problems. Applied and Computational Mathematics. Vol. 6, No. 2, 2017, pp. 88-92. doi: 10.11648/j.acm.20170602.14

Received: February 10, 2016; Accepted: December 29, 2016; Published: April 7, 2017

Abstract: In this paper we have developed an algorithm for n-jobs with m-machine flow shop scheduling problems in order to handle variety of data type than Geleta [3]. In other hands, as in Geleta [3] it main objective is how to obtain optimal sequence of jobs with aim of minimum squared value of lateness.

Keywords: N-jobs M-machine Flow Shop Scheduling Problems, Squared Lateness,

Completion Time and Processing Time Multiple

\section{Introduction}

We extended the work of change [1], Ikram [2] and Geleta [3] to special class of n-jobs m-machine scheduling problems it to handle other data type. The algorithm seems the same with that of Geleta's but it is shifted accordingly to other data type. Still it does not work for all types of data.

As in Geleta [3],throughout this paper, $A_{i j}$ represents the processing time of job $j$ on machine iand the order of machines is fixed and it is $A_{1} \rightarrow A_{2} \rightarrow A_{3} \rightarrow \cdots \rightarrow A_{(m-1)} \rightarrow$ $A_{m}$, with the completion time of job done oni $i^{\text {th }}$ position place is given by

$$
C_{i}=\left[\sum_{j=1}^{i} A_{1 j}\right]+A_{2 i}+A_{3 i}+\cdots+A_{m i}, \text { for } i=1: \mathrm{n}
$$

Problem formulation and assumption

In addition to the assumption of Geleta [3], I want to have only the following:

$$
\begin{gathered}
\operatorname{Max}\left\{A_{1 i}\right\} \leq \operatorname{Min}\left\{A_{2 i}\right\} \\
\operatorname{Max}\left\{A_{2 i}\right\} \leq \operatorname{Min}\left\{A_{3 i}\right\} \\
\operatorname{Max}\left\{A_{3 i}\right\} \leq \operatorname{Min}\left\{A_{4 i}\right\}
\end{gathered}
$$

$$
\operatorname{Max}\left\{A_{(m-1) i}\right\} \leq \operatorname{Min}\left\{A_{m i}\right\}
$$

And if $A_{1 x}<A_{1 y}$, then the following conditions holds true:

$$
\begin{gathered}
A_{1 x} A_{2 y} \leq A_{1 y} A_{2 x} \\
A_{1 x} A_{3 y} \leq A_{1 y} A_{3 x} \\
A_{1 x} A_{4 y} \leq A_{1 y} A_{4 x}
\end{gathered}
$$

$$
A_{1 x} A_{m y} \leq A_{1 y} A_{m x}
$$

Since all jobs spend more amount of time on the last machine, then $d_{j}=\mathrm{k} A_{m j}, j=1: n$, where $k$ is processing time multiple. This is because of the condition (2a) to (2d).

Let $\sigma$ denotes an arbitrary sequence, and $[j]$ represents jobs occupying $j^{\text {th }}$ position of $\sigma$. If $L_{[j]}, C_{[j]}$ and $d_{[j]}$ represents lateness, completion time and assigned due date of the job in the $j^{\text {th }}$, then our objective function is to minimize

$$
L^{2}=\sum_{i=1}^{n} L_{[i]}^{2}=\sum_{i=1}^{n}\left(C_{[i]}-d_{[i]}\right)^{2}
$$

Since the completion time of the $j^{\text {th }}$ for any sequence $\sigma$ is: $C_{[j]}=\left[\sum_{i=1}^{j} A_{[1 i]}\right]+A_{[2 j]}+A_{[3 j]}+\cdots+A_{[m j]}$, for $j=1: \mathrm{n}$ 
and by using $d_{[j]}=\mathrm{k} A_{[1 j]}, j=1:$ nequation (4) becomes:

$$
\left.L^{2}=\sum_{j=1}^{n}\left[\sum_{i=1}^{j} A_{[1 i]}\right]+A_{[2 j]}+A_{[3 j]}+\cdots+A_{[m j]}-\mathrm{k} A_{[1 j]}\right]^{2}
$$

By exactly the same procedure as in Geleta [13], we have the following optimal processing time multiple $\mathrm{k}^{*}$ andoptimal due date:

This is a function of $\mathrm{k}$ and that have to be minimized.

$$
\mathrm{k}^{*}=\frac{\sum_{\mathrm{j}=1}^{\mathrm{n}} \mathrm{A}_{[1 \mathrm{j}]} \sum_{\mathrm{i}=1}^{\mathrm{j}} \mathrm{A}_{[1 \mathrm{i}]}+\sum_{\mathrm{j}=1}^{\mathrm{n}} \mathrm{A}_{[1 \mathrm{j}]} \mathrm{A}_{2 \mathrm{j}}+\sum_{\mathrm{j}=1}^{\mathrm{n}} \mathrm{A}_{[1 \mathrm{j}]} \mathrm{A}_{[3 \mathrm{j} j}+\sum_{\mathrm{j}=1}^{\mathrm{n}} \mathrm{A}_{[1 \mathrm{j}]} \mathrm{A}_{[4 \mathrm{j}]}+\ldots+\sum_{\mathrm{j}=1}^{\mathrm{n}} \mathrm{A}_{[1 \mathrm{j}]} \mathrm{A}_{[\mathrm{mj}]}}{\sum_{\mathrm{j}=1}^{\mathrm{n}}\left[\mathrm{A}_{[1 \mathrm{j}]}\right]^{2}}
$$

Hence, by using the value of $\mathrm{k}^{*}$ we assign the value of due dates of each to be

$$
d_{[j]}=\mathrm{k}^{*} A_{[m j]}, j=1: n
$$

Theorem (Minimization of $L^{2}$ under a certain condition):

Our objective function $L^{2}=\sum_{j=1}^{n}\left[\sum_{i=1}^{j} A_{[1 i]}\right]+A_{[2 j]}+$ $\left.A_{[3 j]}+\cdots+A_{[m j]}-\mathrm{k} A_{[1 j]}\right]^{2}$ can be minimized by applying the rule that job $\mathrm{x}$ should be done before job $\mathrm{y}$ if the following conditions are satisfied:

$$
\begin{gathered}
A_{1 x}<A_{1 y} \\
A_{1 x} A_{2 y} \leq A_{1 y} A_{2 x} \\
A_{1 x} A_{3 y} \leq A_{1 y} A_{3 x}
\end{gathered}
$$

$$
L^{2}=\sum_{j=1}^{n}\left\{\left[\left[\sum_{i=1}^{j} A_{[1 i]}\right]+A_{[2 j]}+A_{[3 j]}+\cdots+A_{[m j]}\right]^{2}+\left[\mathrm{k} A_{[1 j]}\right]^{2}-2 \mathrm{k} A_{[1 j]}\left(\left[\sum_{i=1}^{j} A_{[1 i]}\right]+A_{[2 j]}+A_{[3 j]}+\cdots+A_{[m j]}\right)\right\}
$$

Proof:

Since $\quad, L^{2}=\sum_{j=1}^{n}\left[\left[\sum_{i=1}^{j} A_{[1 i]}\right]+A_{[2 j]}+A_{[3 j]}+\cdots+\right.$ $\left.A_{[m j]}-\mathrm{k} A_{[1 j]}\right]^{2}$, the extending this expression we can obtain that

$$
A_{1 x} A_{4 y} \leq A_{1 y} A_{4 x}
$$

By having re-arrangement of summation inside the bracket we have,

$$
L^{2}=\sum_{j=1}^{n}\left[\left[\sum_{i=1}^{j} A_{[1 i]}\right]+A_{[2 j]}+A_{[3 j]}+\cdots+A_{[m j]}\right]^{2}+\sum_{j=1}^{n}\left[\mathrm{k} A_{[1 j]}\right]^{2}-2 k \sum_{j=1}^{n}\left[A_{[1 j]}\left(\left[\sum_{i=1}^{j} A_{[1 i]}\right]+A_{[2 j]}+A_{[3 j]}+\cdots+A_{[m j]}\right)\right]
$$

From the third term of Equation (7), we obtain:

$$
\sum_{j=1}^{n}\left[A_{[1 j]}\left(\left[\sum_{i=1}^{j} A_{[1 i]}\right]+A_{[2 j]}+A_{[3 j]}+\cdots+A_{[m j]}\right)\right]=
$$

$\sum_{j=1}^{n} A_{[1 j]} \sum_{i=1}^{j} A_{[1 i]}+\sum_{j=1}^{n} A_{[1 j]} A_{2 j}+\sum_{j=1}^{n} A_{[1 j]} A_{[3 j]}+\sum_{j=1}^{n} A_{[1 j]} A_{[4 j]}+\ldots+\sum_{j=1}^{n} A_{[1 j]} A_{[m j]}$, which is constant and independent of the sequence of the jobs change [1].

And also, the middle term $\sum_{j=1}^{n}\left[\mathrm{k} A_{[1 j]}\right]^{2}$ is constant (because of it is the sum of square quantity) and independent of sequence of the jobs.

Now, the remaining thing to prove is that $\sum_{j=1}^{n}\left[\left[\sum_{i=1}^{j} A_{[1 i]}\right]+A_{[2 j]}+A_{[3 j]}+\cdots+A_{[m j]}\right]^{2}$ can be minimized by applying the conditions given above in the theorem?

The answer is yes!

Let $\sigma_{1}$ be a sequence of jobs in which job $\mathrm{x}$ and $\mathrm{y}$ are arranged in a position $\mathrm{k}$ and $\mathrm{k}+1$ respectively, and $\sigma_{2}$ be a sequence of the jobs in which job $\mathrm{x}$ and $\mathrm{y}$ are arranged in apposition of $\mathrm{k}+1$ and $\mathrm{k}$ respectively.

$$
\text { Let } f\left(\sigma_{1}\right)=\sum_{j=1}^{n}\left[\left[\sum_{i=1}^{j} A_{[1 i]}\right]+A_{[2 j]}+A_{[3 j]}+\cdots+A_{[m j]}\right]^{2}
$$

And

$$
f\left(\sigma_{2}\right)=\sum_{j=1}^{n}\left[\left[\sum_{i=1}^{j} A_{[1 i]}\right]+A_{[2 j]}+A_{[3 j]}+\cdots+A_{[m j]}\right]^{2}
$$

Now, by expanding equation (9), we obtain

$$
\begin{aligned}
& f\left(\sigma_{1}\right)=\left[A_{[11]}+A_{[21]}\right.\left.+A_{[31]}+\cdots+A_{[m 1]}\right]^{2}+\left[A_{[11]}+A_{[12]}+A_{[22]}+A_{[32]}+\cdots+A_{[m 2]}\right]^{2}+\left[A_{[11]}+A_{[12]}+A_{[13]}+A_{[23]}+\right. \\
&\left.A_{[33]}+\cdots+A_{[m 3]}\right]^{2}+\left[A_{[11]}+A_{[12]}+A_{[13]}+A_{[14]}+A_{[24]}+A_{[34]}+\cdots+A_{[m 4]}\right]^{2}+\ldots+\left[A_{[11]}+A_{[12]}+A_{[13]}+A_{[14]}+\cdots+\right. \\
&\left.A_{[1(k-1)]}+A_{[1 x]}+A_{[2 x]}+A_{[3 x]}+\cdots+A_{[m x]}\right]^{2}+\left[A_{[11]}+A_{[12]}+A_{[13]}+A_{[14]}+\cdots+A_{[1(k-1)]}+A_{[1 x]}+A_{[1 y]}+A_{[2 y]}+A_{[3 y]}+\right. \\
& \cdots\left.+A_{[m y]}\right]^{2}+\left[A_{[11]}+A_{[12]}+A_{[13]}+A_{[14]}+\cdots+A_{[1(k+2)]}+A_{[2(k+2)]}+A_{[3(k+2)]}+\cdots+A_{[m(k+2)]}\right]^{2}+\ldots \\
&+\left[A_{[11]}+A_{[12]}+A_{[13]}+A_{[14]}+\cdots+A_{[1 n]}+A_{[2 n]}+A_{[3 n]}+\cdots+A_{[m n]}\right]^{2}
\end{aligned}
$$




$$
\begin{gathered}
f\left(\sigma_{2}\right)=\left[A_{[11]}+A_{[21]}+A_{[31]}+\cdots+A_{[m 1]}\right]^{2}+\left[A_{[11]}+A_{[12]}+A_{[22]}+A_{[32]}+\cdots+A_{[m 2]}\right]^{2}+\left[A_{[11]}+A_{[12]}+A_{[13]}+A_{[23]}+A_{[33]}+\cdots+\right. \\
\left.A_{[m 3]}\right]^{2}+\left[A_{[11]}+A_{[12]}+A_{[13]}+A_{[14]}+A_{[24]}+A_{[34]}+\cdots+A_{[m 4]}\right]^{2}+\ldots+\left[A_{[11]}+A_{[12]}+A_{[13]}+A_{[14]}+\cdots+A_{[1(k-1)]}+A_{[2(k-1)]}+\right. \\
\left.A_{[3(k-1)]}+\cdots+A_{[m(k-1)]}\right]^{2}+\left[A_{[11]}+A_{[12]}+A_{[13]}+A_{[14]}+\cdots+A_{[1(k-1)]}+A_{[1 y]}+A_{[2 y]}+A_{[3 y]}+\cdots+\right. \\
\left.A_{[m y]}\right]^{2}+\left[A_{[11]}+A_{[12]}+A_{[13]}+A_{[14]}+\cdots A_{[1(k-1)]}+A_{[1 y]}+A_{[1 x]}+A_{[2 x]}+A_{[3 x]}+\cdots+A_{[m x]}\right]^{2}+\left[A_{[11]}+A_{[12]}+A_{[13]}+\cdots+A_{[1(k+2)]}+\right. \\
\left.A_{[2(k+2)]}+A_{[3(k+2)]}+\cdots+A_{[m(k+2)}\right]^{2}+\ldots+\left[A_{[11]}+A_{[12]}+A_{[13]}+A_{[14]}+\cdots+A_{[1 n]}+A_{[2 n]}+A_{[3 n]}+\cdots+A_{[m n]}\right]^{2}(11) \\
\quad \mathrm{Now}, f\left(\sigma_{1}\right)-f\left(\sigma_{2}\right)=\left[A_{[11]}+A_{[12]}+A_{[13]}+A_{[14]}+\cdots+A_{[1(k-1)]}+A_{[1 x]}+A_{[2 x]}+A_{[3 x]}+\cdots+A_{[m x]}\right]+ \\
{\left[A_{[11]}+A_{[12]}+A_{[13]}+A_{[14]}+\cdots+A_{[1(k-1)]}+A_{[1 x]}+A_{[1 y]}+A_{[2 y]}+A_{[3 y]}+\cdots+A_{[m y]}\right]^{2}} \\
-\left[A_{[11]}+A_{[12]}+A_{[13]}+A_{[14]}+\cdots+A_{[1(k-1)]}+A_{[1 y]}+A_{[2 y]}+A_{[3 y]}+\cdots+A_{[m y]}\right] \\
-\left[A_{[11]}+A_{[12]}+A_{[13]}+A_{[14]}+\cdots A_{[1(k-1)]}+A_{[1 y]}+A_{[1 x]}+A_{[2 x]}+A_{[3 x]}+\cdots+A_{[m x]}\right]^{2}
\end{gathered}
$$

Then by simplifying equation (12) we get

$$
\begin{gathered}
f\left(\sigma_{1}\right)-f\left(\sigma_{2}\right) \\
=A_{[1 x]}\left[A_{[11]}+A_{[12]}+A_{[13]}+A_{[14]}+\cdots+A_{[1(k-1)]}+A_{[1 x]}+A_{[1 y]}+A_{[2 y]}+A_{[3 y]}+\cdots+A_{[m y]}\right]- \\
A_{[1 y]}\left[A_{[11]}+A_{[12]}+A_{[13]}+A_{[14]}+\cdots A_{[1(k-1)]}+A_{[1 y]}+A_{[1 x]}+A_{[2 x]}+A_{[3 x]}+\cdots+A_{[m x]}\right] \\
=A_{[1 x]} A_{[11]}+A_{[1 x]} A_{[12]}+A_{[1 x]} A_{[13]}+\ldots+A_{[1 x]} A_{[1(k-1)]}+A_{[1 x]} A_{[1 x]}+A_{[1 x]} A_{[1 y]}+A_{[1 x]} A_{[2 y]}+A_{[1 x]} A_{[3 y]}+\ldots+A_{[1 x]} A_{[m y]}- \\
A_{[1 y]} A_{[11]-} A_{[1 y]} A_{[12]}-A_{[1 y]} A_{[13]}-A_{[1 y]} A_{[14]}-A_{[1 y]} A_{[1(k-1)]}-A_{[1 y]} A_{[1 x]}-A_{[1 y]} A_{[2 x]}-A_{[1 y]} A_{[3 x]}-A_{[1 y]} A_{[4 x]}-\cdots- \\
A_{[1 y]} A_{[m x]} \\
=\left[A_{[1 x]}\right]^{2}-\left[A_{[1 y]}\right]^{2}+\left(A_{[1 x]}-A_{[1 y]}\right)\left[A_{[11]}+A_{[12]}+A_{[13]}+A_{[14]}+\cdots+A_{[1(k-1)]}\right]+\left[A_{[1 x]} A_{[1 y]}+A_{[1 x]} A_{[2 y]}+A_{[1 x]} A_{[3 y]}+\right. \\
\left.A_{[1 x]} A_{[4 y]}+\cdots+A_{[1 x]} A_{[m y]}-A_{[1 y]} A_{[1 x]}-A_{[1 y]} A_{[2 x]}-A_{[1 y]} A_{[3 x]}-A_{[1 y]} A_{[4 x]}-\cdots-A_{[1 y]} A_{[m x]}\right] \\
=\left[A_{[1 x]}\right]^{2}-\left[A_{[1 y]}\right]^{2}+\left(A_{[1 x]}-A_{[1 y]}\right)\left[A_{[11]}+A_{[12]}+A_{[13]}+A_{[14]}+\cdots+A_{[1(k-1)]}\right]+\left[A_{[1 x]} A_{[1 y]}-A_{[1 y]} A_{[1 x]}\right]+\left[A_{[1 x]} A_{[2 y]}-\right. \\
\left.A_{[1 y]} A_{[2 x]}\right]+\left[A_{[1 x]} A_{[3 y]}-A_{[1 y]} A_{[3 x]}\right]+\left[A_{[1 x]} A_{[4 y]}-A_{[1 y]} A_{[4 x]}\right]+\cdots+\left[A_{[1 x]} A_{[m y]}-A_{[1 y]} A_{[m x]}\right]
\end{gathered}
$$

Now, if $A_{1 x}<A_{1 y}$

$$
\begin{aligned}
& A_{1 x} A_{2 y} \leq A_{1 y} A_{2 x} \\
& A_{1 x} A_{3 y} \leq A_{1 y} A_{3 x} \\
& A_{1 x} A_{4 y} \leq A_{1 y} A_{4 x}
\end{aligned}
$$

$$
A_{1 x} A_{m y} \leq A_{1 y} A_{m x},
$$

Then, $f\left(\sigma_{1}\right)<f\left(\sigma_{2}\right)$.

Thus, the interchanging of job $x$ and $y$ reduces the value of $L^{2}$. Hence, job $x$ should be done before job $y$. Therefore, the conditions stated in the above theorem are satisfied.Now, by repeatedly applying the above rule, $L^{2}$ can be minimized by arranging jobs depending on their processing time on the first machine as S.P.T rule.

\section{Algorithms to Get Optimal Sequence}

Step 1: Verify that the conditions given from (2a)-(2d) and (3a) - (3d). If a1l of these conditions holds true proceed to the next step. Else stop.
Step 2: Determine the values of $k^{*}$ using by the formula (6a).

Step 3: By using shortest processing time rule on the last machine $A_{m}$ determine the optimal sequence of jobs.

Step 4: Finally, find $L^{2}$ for the obtained optimal sequences of jobs.

Recommendation: One can create other algorithm that works for all types of data is my recommendation for other researchers.

\section{Example}

For the following 3-jobs 3-machine flow shop scheduling problem, find the optimal sequence of jobs such that $L^{2}$ is minimum.

\begin{tabular}{llll}
\hline \multirow{2}{*}{ Jobs } & \multicolumn{3}{l}{ Machines } \\
\cline { 2 - 4 } & $\boldsymbol{A}_{\mathbf{1}}$ & $\boldsymbol{A}_{\mathbf{2}}$ & $\boldsymbol{A}_{\mathbf{3}}$ \\
\hline 1 & $A_{11}=3$ & $A_{21}=6$ & $A_{31}=12$ \\
2 & $A_{12}=5$ & $A_{22}=8$ & $A_{32}=10$ \\
3 & $A_{13}=4$ & $A_{23}=7$ & $A_{33}=11$ \\
\hline
\end{tabular}

Solution:

Here, what we have to do is that, according to the above algorithm, we have to find:-

a. $k^{*}$

b. Due-dates of each job.

c. An optimal sequence.

Step 1: 
Clearly, $\max \{3,5,4\}=5<\min \{6,8,7\}=6$

$$
\max \{6,7,8\}=8<\min \{12,10,11\}=10
$$

And also, let say job 1 is $\mathrm{x}$ and job 2 is $\mathrm{y}$.

Then, $A_{1 x} A_{2 y}=(3)(8)=24$

$$
\begin{aligned}
& A_{1 x} A_{3 y}=(3)(10)=30 \\
& A_{1 y} A_{2 x}=(5)(6)=30
\end{aligned}
$$

$$
A_{1 y} A_{3 x}=(5)(12)=60
$$

This implies that

$\left\{\begin{array}{l}A_{1 x} A_{2 y}=(3)(8)=24<A_{1 y} A_{2 x}=30 \\ A_{1 x} A_{3 y}=(3)(10)=30<A_{1 y} A_{3 x}=60\end{array}\right.$

Therefore, all conditions for step 1 are satisfied.

Step 2:

$$
\text { Since, } \mathrm{k}^{*}=\frac{\sum_{\mathrm{j}=1}^{\mathrm{n}} \mathrm{A}_{[1 \mathrm{j}]} \sum_{\mathrm{i}=1}^{\mathrm{j}} \mathrm{A}_{[1 \mathrm{i}]}+\sum_{\mathrm{j}=1}^{\mathrm{n}} \mathrm{A}_{[1 \mathrm{j}]} \mathrm{A}_{2 \mathrm{j}}+\sum_{\mathrm{j}=1}^{\mathrm{n}} \mathrm{A}_{[1 \mathrm{j}]} \mathrm{A}_{[3 \mathrm{j}]}+\sum_{\mathrm{j}=1}^{\mathrm{n}} \mathrm{A}_{[1 \mathrm{j}]} \mathrm{A}_{[4 \mathrm{j}]}+\ldots+\sum_{\mathrm{j}=1}^{\mathrm{n}} \mathrm{A}_{[1 \mathrm{j}]} \mathrm{A}_{[m \mathrm{~m}]}}{\sum_{\mathrm{j}=1}^{\mathrm{n}}\left[\mathrm{A}_{[1 \mathrm{j}]}\right]^{2}} \ldots
$$

Then, because of our problem is for $\mathrm{n}=3=\mathrm{m}, \mathrm{k}^{*}$ becomes,

$$
\begin{aligned}
& \mathrm{k}^{*}=\frac{\sum_{\mathrm{j}=1}^{3} \mathrm{~A}_{[1 \mathrm{j}]} \sum_{\mathrm{i}=1}^{\mathrm{j}} \mathrm{A}_{[1 \mathrm{i}]}+\sum_{\mathrm{j}=1}^{3} \mathrm{~A}_{[1 \mathrm{j}]} \mathrm{A}_{2 \mathrm{j}}+\sum_{\mathrm{j}=1}^{3} \mathrm{~A}_{[1 \mathrm{j}]} \mathrm{A}_{[3 \mathrm{j}]}}{\sum_{\mathrm{j}=1}^{3}\left[\mathrm{~A}_{[1 \mathrm{j}]}\right]^{2}} \\
& =\frac{A_{11}\left(A_{11}\right)+\left(A_{11}+A_{12}\right)\left(A_{11}+A_{12}\right)+\left(A_{11}+A_{12} A_{13}\right)\left(A_{11}+A_{12} A_{13}\right)+A_{11} A_{21}+A_{12} A_{22}+A_{13} A_{23}+A_{11} A_{31}+A_{12} A_{32}+A_{11} A_{33}}{A_{11}{ }^{2}+A_{12}{ }^{2}+A_{13}{ }^{2}} \\
& =\frac{(3)(3)+(3+5)(3+5)+(3+5+4)(3+5+4)+(3)(6)+(5)(8)+(4)(7)+(3)(12)+(5)(10)+(3)(11)}{(3)^{2}+(5)^{2}+(4)^{2}}=\frac{424}{50} \\
& =8.48
\end{aligned}
$$

Now, by using this values of $\mathrm{k}^{*}$ we can assign the due dates of each job as follows:

\begin{tabular}{lll}
\hline Jobs & Machine $\boldsymbol{A}_{\mathbf{3}}$ & Due-date $\left(\boldsymbol{d}_{[\boldsymbol{j}]}=\mathbf{k}^{*} \boldsymbol{A}_{[\mathbf{3 j}]}\right)$ \\
\hline 1 & 12 & 101.76 \\
2 & 10 & 84.8 \\
3 & 11 & 93.28 \\
\hline
\end{tabular}

Step 3:

As indicated in the above algorithm, we arrange jobs as per shortest processing time rule on machine $3\left(A_{3}\right)$. And also, the same result we obtain, if we arrange jobs by earliest due date rule.

Therefore, by using both of them (one of them is enough), we obtain the optimal sequence of jobs 2-3-1.

Step 4:

Determination of $L^{2}$. Here, $L^{2}$ is listed in the following table for all possible sequences of jobs we have.

\begin{tabular}{lll}
\hline $\begin{array}{l}\text { Sequences of } \\
\text { jobs }(\boldsymbol{\sigma})\end{array}$ & $\begin{array}{l}\text { Processing time } \\
\text { multiple } \mathbf{k}^{*}\end{array}$ & $\begin{array}{l}\text { Squared value of } \\
\text { lateness } \boldsymbol{L}^{\mathbf{2}}\end{array}$ \\
\hline $1-2-3$ & 8.48 & 13983.976 \\
$1-3-2$ & 8.48 & 14187.376 \\
$3-1-2$ & 8.48 & 13975.976 \\
$3-2-1$ & 8.48 & 13687.776 \\
$2-3-1$ & 8.48 & 13361.776 \\
$2-1-3$ & 8.48 & 11254.736 \\
\hline
\end{tabular}

Note that, $L^{2}$ is calculated by

\begin{tabular}{|c|c|c|c|}
\hline \multirow{2}{*}{ Jobs } & \multicolumn{3}{|c|}{ Machines } \\
\hline & $A_{1}$ & $A_{2}$ & $A_{3}$ \\
\hline 1 & $A_{11}=3$ & $A_{21}=6$ & $A_{31}=12$ \\
\hline 3 & $A_{12}=4$ & $A_{22}=7$ & $A_{32}=11$ \\
\hline 2 & $A_{13}=5$ & $A_{23}=8$ & $A_{33}=10$ \\
\hline \multicolumn{4}{|c|}{$\begin{array}{l}L^{2}=(3+6+12-(8.48) 12)^{2}+(3+4+7+11- \\
(8.48) 11)^{2}+(3+4+5+8+10-(8.48) 10)^{2}\end{array}$} \\
\hline \multicolumn{4}{|c|}{$=(21-101.76)^{2}+(25-93.28)^{2}+(30-84.8)^{2}$} \\
\hline \multicolumn{4}{|c|}{$=(-80.76)^{2}+(-68.28)^{2}+(-54.8)^{2}$} \\
\hline \multicolumn{4}{|c|}{$=6522.1776+4662.1584+3003.04$} \\
\hline & & .376 & \\
\hline
\end{tabular}

$L^{2}=\sum_{j=1}^{n}\left[\sum_{i=1}^{j} A_{[1 i]}\right]+A_{[2 j]}+A_{[3 j]}+\cdots+A_{[m j]}-$ $\left.\mathrm{k}^{*} A_{[3 j]}\right]^{2}$, because of $n=m=3$ for our problem, then it becomes

$$
\begin{gathered}
\left.L^{2}=\sum_{j=1}^{3}\left[\sum_{i=1}^{j} A_{[1 i]}\right]+A_{[2 j]}+A_{[3 j]}-\mathrm{k}^{*} A_{[3 j]}\right]^{2} \\
=\left(A_{11}+A_{21+} A_{31}-\mathrm{k}^{*} A_{11}\right)^{2}+\left(A_{11}+A_{12+} A_{22+} A_{32}-\right. \\
\left.\mathrm{k}^{*} A_{32}\right)^{2}+\left(A_{11}+A_{12+} A_{13+} A_{23}+A_{33}-\mathrm{k}^{*} A_{33}\right)^{2}
\end{gathered}
$$

For instance, for the sequence of jobs2-3-1:-

\begin{tabular}{llll}
\hline \multirow{2}{*}{ Jobs } & \multicolumn{2}{l}{ Machines } & \\
\cline { 2 - 4 } & $\boldsymbol{A}_{\mathbf{1}}$ & $\boldsymbol{A}_{\mathbf{2}}$ & $\boldsymbol{A}_{\mathbf{3}}$ \\
\hline 2 & $A_{11}=5$ & $A_{21}=8$ & $A_{31}=10$ \\
3 & $A_{12}=4$ & $A_{22}=7$ & $A_{32}=11$ \\
1 & $A_{13}=3$ & $A_{23}=6$ & $A_{33}=12$ \\
\hline
\end{tabular}

$$
\begin{gathered}
L^{2}=(5+8+10-(8.48) 10)^{2}+(5+4+7+11- \\
(8.48) 11)^{2}+(5+4+3+6+12-(8.48) 12)^{2} \\
=(23-84.8)^{2}+(27-93.28)^{2}+(30-101.76)^{2} \\
=(-61.8)^{2}+(-66.28)^{2}+(-71.76)^{2} \\
=3819.24+4393.0384+5149.4976 \\
=13361.776
\end{gathered}
$$

Similarly, for the sequence of jobs 1-3-2 we have,

For the sequence of jobs1-2-3 we have, 


\begin{tabular}{|c|c|c|c|}
\hline \multirow{2}{*}{ Jobs } & \multicolumn{3}{|c|}{ Machines } \\
\hline & $A_{1}$ & $A_{2}$ & $A_{3}$ \\
\hline 1 & $A_{11}=3$ & $A_{21}=6$ & $A_{31}=12$ \\
\hline 2 & $A_{12}=5$ & $A_{22}=8$ & $A_{32}=10$ \\
\hline 3 & $A_{13}=4$ & $A_{23}=7$ & $A_{33}=11$ \\
\hline
\end{tabular}

$L^{2}=(3+6+12-(8.48) 12)^{2}+(3+5+8+10-$ $(8.48) 10)^{2}+(3+5+4+7+11-(8.48) 11)^{2}$

$$
\begin{gathered}
=(21-101.76)^{2}+(26-84.8)^{2}+(30-93.28)^{2} \\
=(-80.76)^{2}+(-58.8)^{2}+(-63.28)^{2} \\
=6522.1776+3457.44+4004.3584
\end{gathered}
$$$$
=13983.976
$$

For the sequence of jobs 2-1-3 we have,

\begin{tabular}{llll}
\hline \multirow{2}{*}{ Jobs } & \multicolumn{3}{l}{ Machines } \\
\cline { 2 - 4 } & $\boldsymbol{A}_{\mathbf{1}}$ & $\boldsymbol{A}_{\mathbf{2}}$ & $\boldsymbol{A}_{\mathbf{3}}$ \\
\hline 2 & $A_{11}=5$ & $A_{21}=8$ & $A_{31}=10$ \\
1 & $A_{12}=3$ & $A_{22}=6$ & $A_{32}=12$ \\
3 & $A_{13}=4$ & $A_{23}=7$ & $A_{33}=11$ \\
\hline
\end{tabular}

$L^{2}=(5+8+10-(8.48) 10)^{2}+(5+3+6+12-$

$(8.48) 12)^{2}+(5+3+4+7+11-(8.48) 11)^{2}$

$$
\begin{gathered}
=(23-84.8)^{2}+(31-101.76)^{2}+(44-93.28)^{2} \\
=(-61.8)^{2}+(-70.76)^{2}+(-49.28)^{2} \\
=3819.24+5006.9776+2428.5184
\end{gathered}
$$$$
=11254.736
$$

For the sequence of jobs 3-1-2 we have,

\begin{tabular}{llll}
\hline \multirow{2}{*}{ Jobs } & \multicolumn{3}{l}{ Machines } \\
\cline { 2 - 4 } & $\boldsymbol{A}_{\mathbf{1}}$ & $\boldsymbol{A}_{\mathbf{2}}$ & $\boldsymbol{A}_{\mathbf{3}}$ \\
\hline 3 & $A_{11}=4$ & $A_{21}=7$ & $A_{31}=11$ \\
1 & $A_{12}=3$ & $A_{22}=6$ & $A_{32}=12$ \\
2 & $A_{13}=5$ & $A_{23}=8$ & $A_{33}=10$ \\
\hline
\end{tabular}

\begin{tabular}{|c|c|c|c|}
\hline \multirow{2}{*}{ Jobs } & \multicolumn{3}{|c|}{ Machines } \\
\hline & $A_{1}$ & $A_{2}$ & $A_{3}$ \\
\hline 3 & $A_{11}=4$ & $A_{21}=7$ & $A_{31}=11$ \\
\hline 2 & $A_{12}=5$ & $A_{22}=8$ & $A_{32}=10$ \\
\hline 1 & $A_{13}=3$ & $A_{23}=6$ & $A_{33}=12$ \\
\hline
\end{tabular}

$$
L^{2}=(4+7+11-(8.48) 11)^{2}+(4+3+6+12-
$$$$
(8.48) 12)^{2}+(4+3+5+8+10-(8.48) 10)^{2}
$$

$$
\begin{gathered}
=(22-93.28)^{2}+(25-101.76)^{2}+(30-84.8)^{2} \\
=(-71.28)^{2}+(-76.76)^{2}+(-54.8)^{2} \\
=5080.8384+5892.0976+3003.04
\end{gathered}
$$

$$
=13975.976
$$

For the sequence of jobs3-2-1 we have,

$L^{2}=(4+7+11-(8.48) 11)^{2}+(4+5+8+10-$ $(8.48) 10)^{2}+(4+5+3+6+12-(8.48) 12)^{2}$ $=(22-93.28)^{2}+(26-84.8)^{2}+(30-101.76)^{2}$

$$
\begin{gathered}
=(-71.28)^{2}+(-58.8)^{2}+(-71.76)^{2} \\
=5080.8384+3457.44+5149.4976 \\
=13687.776
\end{gathered}
$$

Now, from the above table the optimal sequence of the given jobs is 2-3-1 with $L^{2}=11254.736$

$($ Jobx $=2$ isdonejoby $=1)$. This completes our example.

\section{References}

[1] Chenge, T. C. E., "Optimal due date determination and scheduling of n- jobs on a single machine", Journal of the Operation Research society 35, pp.433-437, 1984.

[2] Ikram, M.," A note on minimization of lateness Cost Function and Determination of optimal Due -date in two machine problem," Pure Applied Mathematika sciences, Vol. XXIII, 12, March 1986.

[3] Geleta Tadele Mohammed. New Algorithm for N-jobs on Mmachine Flow Shop Scheduling Problems. Applied and Computational Mathematics Journal. Vol. 5, No. 1, 2016, pp. 1-9. doi: $10.11648 /$ j.acm.20160501.11

[4] Johnson, S. M. "Optimal two and three stage production schedules with setup times included." Naval Research Logistics Quarterly, Vol I, 61-68, 1954.

[5] [5]Jackson, J.R.," An extension of Johnson's results on lot scheduling", Naval Research Logistics Quarterly, Vol 3, pp.201-2003, 1956.

[6] Ibrahim, M. A., "Algorithms for Sequencing and Scheduling," Industrial Engineering Department, College of Engineering, King saud University, Riyadh, Saudi Arabia.

[7] Osman, M.R., Ismail, N., Zairian, M.R.M, Yusuf, R.M., Sapuan, S. M." sheet metal Fabrication Scheduling Using Selective Performance Measure and priority Dispatching Rule," International Journal of Engineering and Technology, Vol.1 (1), pp.74-83, 2004.

[8] Taylor, F.W., "Shop Management", Harper and Bros, New York, 1911.

[9] Herrmann, Jeffrey. W "A history of production Scheduling” in Handbook of Production Scheduling, Springer, New York, 2006.

[10] Dudek, R. A., S. S. Panwalkar, and M.L. Smith,” The lessons for flow shop scheduling Research," Operation Research, Vol 40(1), pp.7-13, 1993.

[11] Conway, R.W., Maxwell, W.L., and Miller, L.W." Theory of Scheduling," Addison-Wesley Publishing Company, Massachusetts, 1967.

[12] Miloš Šeda "Mathematical Models of Flow Shop and Job Shop Scheduling Problems", International Journal of Applied Mathematics and Computer Sciences Volume 4 Number 4.

[13] Kerem Bülbül Philip Kaminsky, Candace Yano,” Flow Shop Scheduling with Earliness, Tardiness, and Intermediate Inventory Holding Costs", Industrial Engineering and Operations Research, University of California, Berkeley, California 94720-1777. 